\title{
Prion disease risk uncertainties associated with urine-derived and recombinant fertility drugs
}

\author{
Neil R. Cashman* \\ Department of Neurology, \\ Faculty of Medicine, \\ Vancouver Coastal Health, \\ University of British Columbia, Canada \\ Email: Neil.Cashman@vch.ca \\ *Corresponding author
}

\section{Michael G. Tyshenko}

McLaughlin Centre for Population Health Risk Assessment, University of Ottawa,

600 Peter Morand, Ottawa,

ON, K1G 3Z7, Canada

Email: mtyshenk@uottawa.ca

\section{Ricky Cheung}

Health Canada, 100 Eglantine Driveway,

Ottawa, Ontario K1A 0K9, Canada

Email: ricky.cheung@utoronto.ca

\section{Willy Aspinall}

\author{
Aspinall and Associates, \\ Cleveland House, \\ Tisbury, UK \\ and \\ Cabot Institute and School of Earth Sciences, \\ Bristol University, \\ Bristol, UK \\ Email: Willy.Aspinall@bristol.ac.uk
}

\section{Michelle Wong}

Office of Research, Faculty of Medicine, University of British Columbia, 317-2194 Health Sciences Mall, Vancouver, BC, V6T 1Z3, Canada Email: michelle.wong@ubc.ca 


\title{
Daniel Krewski
}

McLaughlin Centre for Population Health Risk Assessment, Institute of Population Health, University of Ottawa, 600 Peter Morand, Ottawa, Ontario, K1G 3Z7, Canada and

Department of Epidemiology and Community Medicine, Faculty of Medicine,

University of Ottawa, 451 Smyth Road, Ottawa, Ontario, K1H 8M5, Canada

Email: dkrewski@uottawa.ca and

Risk Sciences International, 55 Metcalfe Street, Suite 700, Ottawa, ON K1P 6L5, Canada

\begin{abstract}
The detection of prion protein in widely used urine-derived fertility drugs has raised the possibility that prions from urine donors with (asymptomatic) prion disease could be present in these drugs. A high level of uncertainty exists regarding this issue. An international expert panel provided judgments related to prion disease transmission through fertility drug use in a structured expert elicitation. The elicitation gauged expert judgements about the uncertainty surrounding potential prion disease risks associated with urine-derived fertility drugs and emphasised the scientific ambiguity surrounding disease transmission risk factors associated with urine-derived fertility drugs. Group aggregated responses indicate that the theoretical risk of prion disease transmission with urine-derived fertility drugs was judged to be very low. The experts judged recombinant fertility drugs produced with bovine serum to possess 10 -fold lower risk compared to urine-derived fertility drugs. Fertility drugs made without fetal bovine serum were judged to present a risk approximately 1,200 times lower compared to urine-derived counterparts. This elicitation indicates recombinant fertility drugs carry relatively less risk than urine-derived fertility drugs. However, the associated uncertainties are significant and pro-active surveillance of possible new routes of transmission of human prion disease warrants consideration of new scientific data as it becomes available.
\end{abstract}

Keywords: prion disease; variant Creutzfeldt-Jakob disease; urine; recombinant; fertility; transmission; expert elicitation; uncertainties.

Reference to this paper should be made as follows: Cashman, N.R., Tyshenko, M.G., Cheung, R., Aspinall, W., Wong, M. and Krewski, D. (2019) 'Prion disease risk uncertainties associated with urine-derived and recombinant fertility drugs', Int. J. Risk Assessment and Management, Vol. 22, No. 2, pp.109-127.

Biographical notes: Neil R. Cashman is a neurologist-neuroscientist working in the Neurodegeneration and Neuroimmunology. His special areas of work are the motor neuron diseases, particularly amyotrophic lateral sclerosis and the amyloid encephalopathies, including prion illnesses and Alzheimer's disease. $\mathrm{He}$ is a Professor of Medicine at the University of British Columbia, where he 
holds the Canada Research Chair in Neurodegeneration and Protein Misfolding Diseases. He is the Founder and Chief Scientific Officer of the ProMIS Neurosciences in Toronto. Special honours include the Jonas Salk Prize for 'A lifetime of outstanding contributions to basic biomedical research', his Tier 1 Canada Research Chair in at the UBC (2005-2018), election to the Canadian Academy of Health Sciences in 2008 and Genome BC award for Scientific Excellence in 2012.

Michael G. Tyshenko is a Research Associate at the University of Ottawa. He specialises in interdisciplinary studies combining science, health policy, communication, risk management and impacts on society. Research areas include: emerging health risk issues, eHealth-mHealth development, biotechnology, zoonotic disease management and burden of disease studies.

Ricky Cheung is a clinical evaluator at the Health Canada. His academic background includes a PhD in Pharmacology, Post-Doctoral Training in Drug Discovery Research and Interdisciplinary Training in Population Health Risk Assessment and Management. His research interests include human health risk assessment issues relating to pharmaceutical products.

Willy Aspinall is a consulting Chartered Scientist and Chartered Geologist and Cabot Professor in Natural Hazards and Risk Science at the University of Bristol UK. His main interests are in risk assessment, volcanology and seismology and the formalised use of expert judgement in decision-making for low probability, high consequence events in circumstances of scientific uncertainty. He specialises in facilitating expert elicitations in medical, climate, Earth science and other science-based fields. He was awarded the 2012 William Smith Medal of the Geological Society of London for excellence in contributions to applied geoscience and was co-recipient with Bristol University colleagues of the 2014 Lloyd's of London Science of Risk Prize for Natural Hazards and the 2015 The Queen's Anniversary Prize for Higher and Further Education.

Michelle Wong is the Senior Director at the Research for the Faculty of Medicine at the University of British Columbia. In this capacity, she leads the creation and implementation of high profile strategic and key pan-institution initiatives, in addition to portfolio management and program delivery capability to enable the faculty and university to implement a broad range of enterprise-wide strategic initiatives. Holding various senior leadership roles related to research, business development, and strategic initiatives over the last ten years, she has received awards and currently serves on many board of directors and committees.

Daniel Krewski is a Professor in the School of Epidemiology, Public Health and Preventive Medicine at the University of Ottawa, where he also serves as Scientific Director of the McLaughlin Centre for Population Health Risk Assessment. He also serves as Chief Risk Scientist for Risk Sciences International, which works in partnership with the University of Ottawa. His research interests include epidemiology, biostatistics, risk assessment and risk management. He is a fellow of the Society for Risk Analysis, the American Statistical Association and a National Affiliate of the US National Academy of Sciences. He holds the Natural Sciences and Engineering Research Council of Canada Chair in Risk Science at the University of Ottawa. 


\section{Introduction}

Creutzfeldt-Jakob disease (CJD) is a human neurodegenerative disorder that is currently incurable and invariably fatal. CJD can be classified into four forms:

1 iatrogenic

2 familial

3 sporadic

4 variant (Sikorska et al., 2012).

Evidence suggests variant CJD (VCJD) may occur due to transmission of prions from cattle to human through the consumption of contaminated meat products derived from affected cattle in the food chain (Brown, 2001; Bruce, 2000; Chen et al., 2013).

Over the last two decades the scientific understanding of the etiology of prion diseases has advanced considerably yielding a better understanding of how these diseases are transmitted both within and between species through contact with infectious prions (Sikorska et al., 2012; Hueston, 2013; Gough and Maddison, 2010). Prion diseases are postulated to be produced by misfolding of a benign, ubiquitously expressed cellular prion protein $\left(\mathrm{PrP}^{\mathrm{C}}\right)$ into a distinct pathological conformation termed scrapie prion protein $\left(\mathrm{PrP}^{\mathrm{Sc}}\right)$, which is considered the infectious and amplifiable disease agent (Prusiner, 1998).

Prion proteins were found to be excreted from infected animals through multiple routes including skin, feces, urine, saliva, blood, milk, placenta and nasal secretions (Gough and Maddison, 2010). Potential routes of transmission for developing human prion disease include surgical procedures, blood transfusion, injection of contaminated cadaveric human growth hormone and consumption of contaminated meat products (Chen et al., 2013; Hamaguchi et al., 2009; Puopolo et al., 2011; Croes et al., 2002).

Van Dorsselaer et al. (2011) reported the detection of normal prion protein in urine-derived fertility drugs using advanced proteomics techniques. Urine-derived drugs are comprised of human menopausal gonadotropins (hMG) or menotropins extracted from female urine that contains follicle stimulating hormone (FSH), luteinising hormone (LH) (e.g., pergonal, menogon) and low levels of human chorionic gonadotropin (hCG). FSH and LH are found in high amounts in urine of post-menopausal women. hMG is derived as a purified extract from the urine of post-menopausal women and is a powerful and effective drug that stimulates the ovaries to produce multiple follicles. Injections of $\mathrm{hMG}$ are given repeatedly over several days in the first half of the cycle and induce ovulation in approximately $75 \%-85 \%$ of patients attempting to conceive as a way to overcome infertility problems (Van De Weijer et al., 2003; Wolfenson et al., 2005).

The detection of prion protein in urine-derived fertility drugs raises the possibility of a new route of transmission of human prion disease, which could conceivably occur if infectious prions were present in urine samples donated by an asymptomatic CJD or vCJD case (Reichl et al., 2002). This route of transmission remains a theoretical possibility as there are no apparent documented cases of prion disease reported following the use of urine-derived gonadotropins. To evaluate the likelihood of transmission of 
human prion disease through the use of urine-derived fertility drugs in the absence of data on transmission rates, a group of international experts on prion disease was consulted to obtain their views on this issue. This consultation was undertaken in the form of a structured expert elicitation, a technique that has been successfully previously used to evaluate uncertainty associated with a wide range of issues (Cooke and Goossens, 2008; Aspinall, 2010) including prion disease risk uncertainties (Tyshenko et al., 2011; 2012).

Expert elicitations of this type may provide useful information regarding potential risks, for which conventional assessment is not possible due to the lack of current scientific data. The findings may be used to make informed decisions regarding risk management options for mitigating prion disease risks related to treatment with urine-derived fertility drugs. The expert elicitation process has the advantage that it provides a synthesis of science-based support for policy responses allowing regulators to conduct risk assessments under a precautionary paradigm to address emerging threats at an early stage, despite the absence of definitive scientific evidence.

The overall goal of the expert elicitation described in this paper was to assess the likelihood that cases of human prion disease may occur through the therapeutic use of urine-derived fertility drugs contaminated with infectious prions.

\section{Methods}

\subsection{Expert elicitation}

The core protocol for the expert elicitation involves two sets of questions. The experts were first asked to answer a series of seed questions related to prion disease for which values are, in principle, knowable, based on existing data. However, the seed items were chosen by an independent facilitator, in such a way that while the experts are not expected to know precise values they are expected to 'capture' these values quantitatively by providing judicious estimates of medians and related $90 \%$ credible interval quantiles.

The experts were then asked to answer, again with median estimate and credible range, a series of target questions related to the objectives of the elicitation; these values are subject to greater uncertainty because of the lack of data relating to the specific issues. The seed questions were used to calibrate expert uncertainty judgement in the field of prion disease. Individual responses to the target questions were weighted by the expert's performance on the seed questions, leading to an aggregated group judgement for each of the target questions, along with an assigned quantitative indicator of uncertainty surrounding that response (both weighted and unweighted analysis of the responses from the experts to the target questions are typically presented in elicitations of this type).

The authors developed 26 target questions for the present elicitation exercise. The questions were grouped into four categories:

1 The prevalence of asymptomatic prion disease in the general population (questions $1-16)$.

2 The presence of infectious prions in the urine pools used in the manufacture of fertility drugs (questions 17-18). 
3 Transmission of prion disease through the use of urine-derived fertility drugs (questions 19-22).

4 The relative risk of transmitting prion disease through the use of urine-derived and recombinant fertility drugs (questions 23-26).

The expert elicitation on uncertainties associated with risks and benefits of treatment with urine-derived and recombinant fertility drugs was held on October 8-9, 2011, at the International Prevention Research Institute (IPRI) in Lyon, France. More than 20 people attended the elicitation workshop, including seven experts who participated actively in the structured elicitation exercise for quantifying uncertainties relating to the transmission of prion diseases via administration of fertility drugs to women. One of the original eight experts did not complete all the seed questions, so the responses of that expert to the target questions could not be used in the overall elicitation analysis. The persons participating in the elicitation were selected for their internationally recognised expertise with respect to prion disease risk issues and for the absence or declaration of conflict of interest in providing their judgements regarding the issues.

Each expert was asked to provide responses in the form of two quantiles defining the 5th and 95th percentile values for their judged uncertainty distribution for each question (i.e., $90 \%$ credible interval). They also provided a 50 th percentile quantity, to represent their judgment of the appropriate central value for the question. The collective response of the seven experts was calculated in two ways:

1 unweighted, treating all experts' judgements as equally valid

2 performance weighted, taking into account the performance of the experts on the seed questions.

The results of the expert elicitation were analysed using the Classical Model for combining expert opinions via the EXCALIBUR software package, which is described in detail in previous publications (Cooke and Goosens, 2008; Cooke, 1991). The EXCALIBUR software package can be freely downloaded at: http://www.expertsinuncertainty.net/Publications/Excalibur/tabid/4386/Default.aspx.

\subsection{Strategy ranking elicitation}

In a second exercise, experts were asked to rank different strategies for managing risks of development of prion disease from taking fertility drugs, by suggesting what weights could be distributed across the strategies with the ultimate the purpose of ranking alternative risk management approaches. These weights were then used as a basis for comparing the relative effectiveness of each strategy. In this exercise, eight risk management strategies were identified by the authors before the elicitation workshop; and two additional strategies were added to the list following discussion by the expert group during the workshop. The final list of 10 potential strategies for managing the risk of developing prion disease from treatment with urine-derived fertility drugs were:

1 donor selection/sourcing from countries without CJD

2 donor selection/sourcing based on time spent in the UK 
3 donor selection/sourcing based on blood transfusion, neurosurgery, or treatment with growth hormone

4 implementing a validated process for sterilisation of donated urine pools to eliminate infectious prions

5 testing for PrP in urine pools used to manufacture gonadotropins

6 testing for PrP in the final product

7 banning the use of urine-derived fertility products

8 banning the use of recombinant fertility products

9 screening for the presence of $\mathrm{PrP}^{\mathrm{Sc}}$ in donated urine using a sensitive, validated test

10 testing for $\mathrm{PrP}^{\mathrm{Sc}}$ using a sensitive, validated test in the final product.

The effectiveness of the risk management strategies was classified into five categories:

1 extremely effective

2 very effective

3 moderately effective

4 minimally effective

5 not at all effective.

In order to allow individual experts to express some degree of uncertainty as to which particular management strategy should be assigned, each expert was told they had a mythical $€ 10$ to invest on the options, with minimum stake size of $€ 1$. The experts endowed their stakes - as each judged appropriate - on the five effectiveness categories for the ten strategies and the responses of the expert panel were conflated and normalised to 100 . In this way, indicative weights were ascribed to the alternatives, on the strength of the expert judgments.

\section{Results}

\subsection{Expert elicitation}

The individual expert responses to the 26 target questions are shown in Figures S1-S26, provided in the supplementary material (available at: https://dataverse.harvard.edu/ dataset.xhtml?persistentId=doi\%3A10.7910\%2FDVN\%2FREVVTB\#). For reach expert, the 5th and 95th percentiles denote their reported credible range, with the 50th percentile representing their central value judgment in response to these questions (the unweighted and weighted combinations of their individual opinions are designated by 'Equal' and 'Perf' in the figures). The performance weighted median and $90 \%$ credible interval for the 26 target questions are summarised in Table 1. 
Table 1 Median performance-weighted responses and $90 \%$ credible intervals provided by the experts to target questions $1-26$

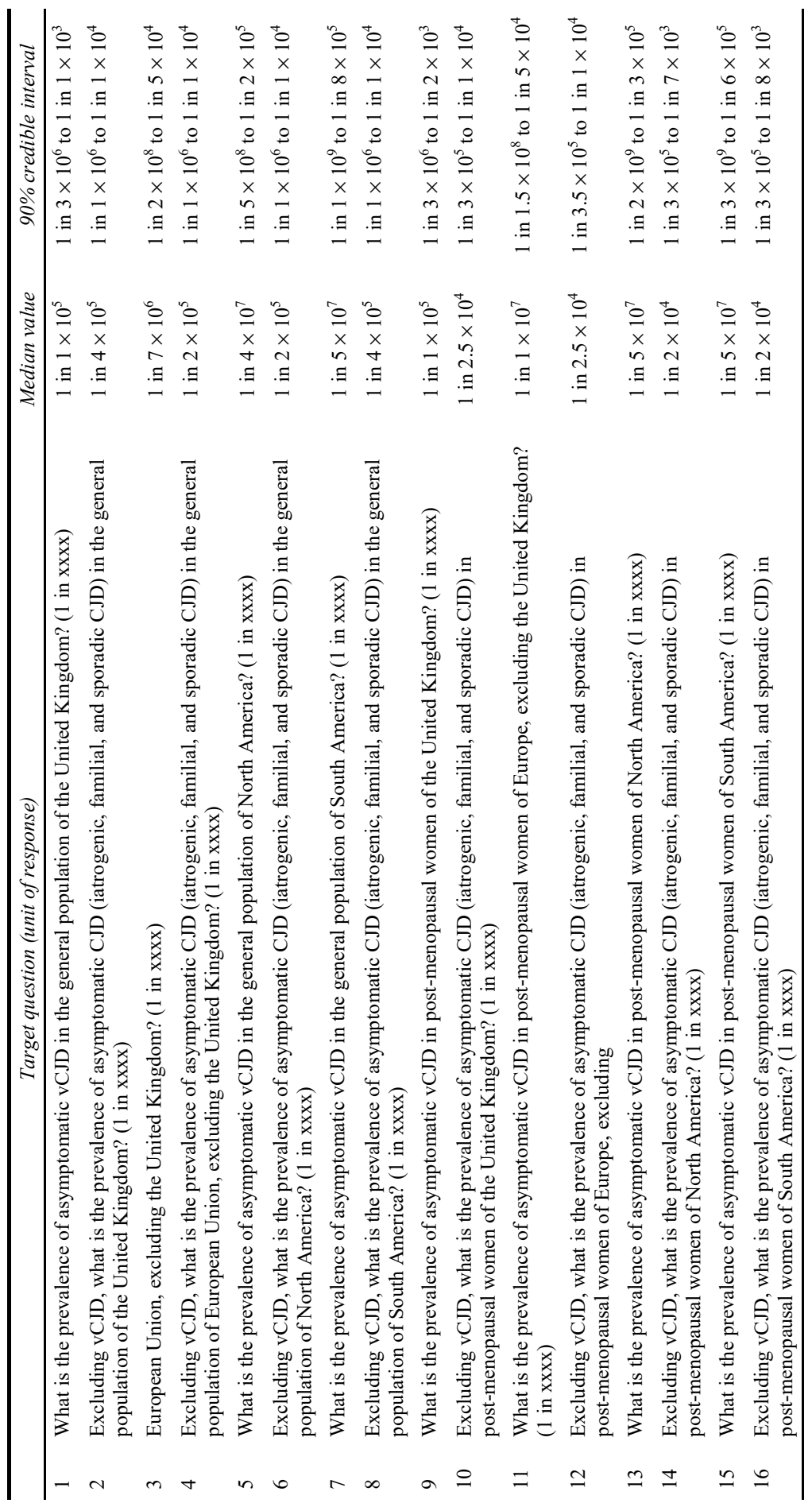


Table 1 Median performance-weighted responses and $90 \%$ credible intervals provided by the experts to target questions 1-26 (continued)

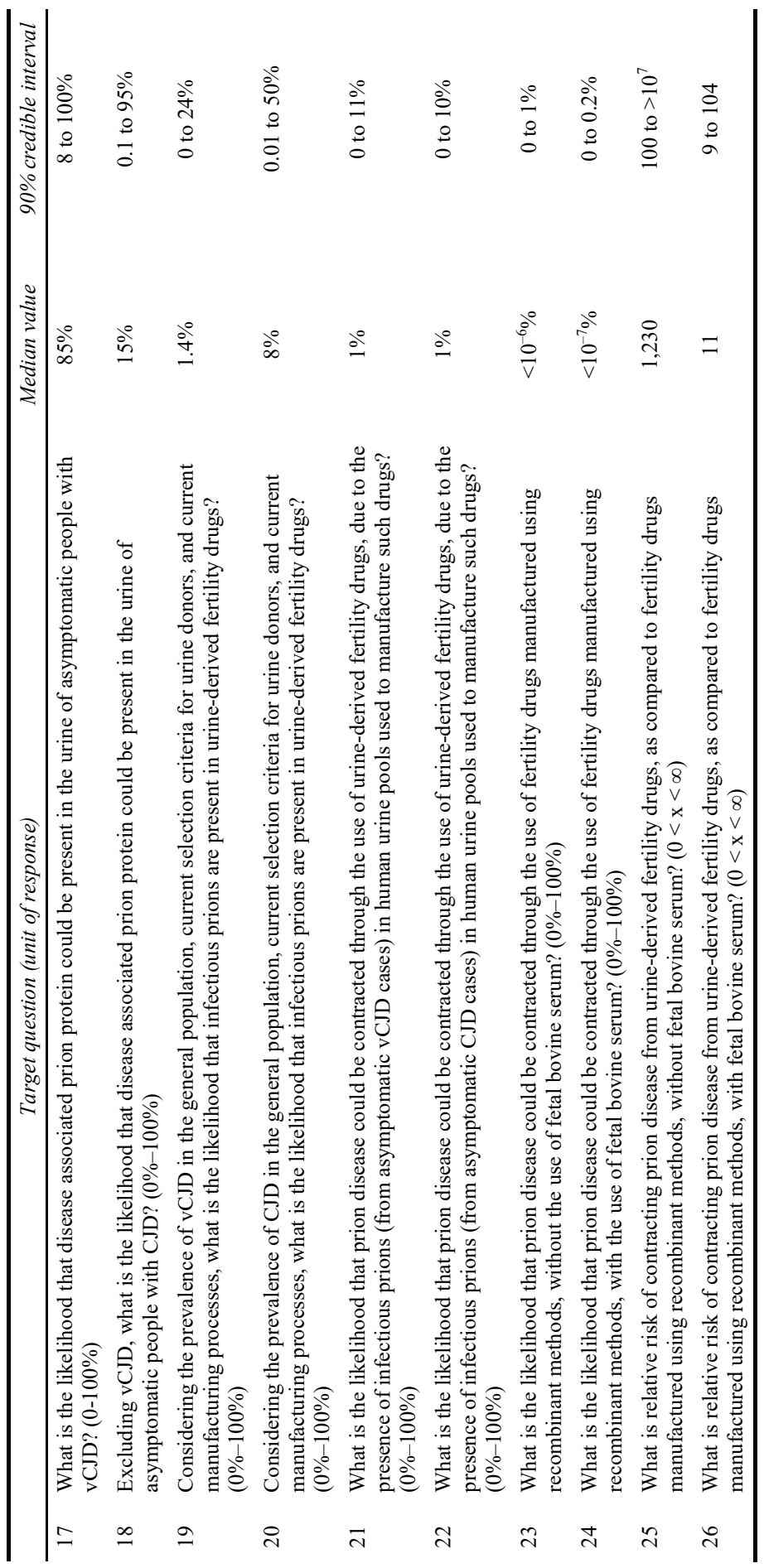




\subsection{Prevalence of asymptomatic prion disease in the general population}

Target questions 1-8 asked the experts to estimate the current prevalence of asymptomatic VCJD and CJD in the general population from four different regions: the UK, European Union excluding the UK, North America and South America. The median prevalence estimates for CJD estimates were similar in these four regions, in the range of 1 in 200,000 to 1 in 400,000. However, prevalence estimates for asymptomatic vCJD reflected the known distribution of reported cases, being highest in the United Kingdom (median prevalence of asymptomatic VCJD was estimated at 1 in 100,000), followed by a smaller number of cases within the European Union (median asymptomatic vCJD estimate of 1 in $7 \times 10^{6}$ ) and even fewer cases reported in North and South America (median asymptomatic vCJD estimates of 1 in $4 \times 10^{7}$ and 1 in $5 \times 10^{7}$ respectively).

Target questions 9-16 asked the experts to estimate the current prevalence of asymptomatic VCJD and CJD in post-menopausal women in these four regions The median prevalence estimates provided by the experts showed a similar pattern to those given for the general population in Table 1 .

\subsection{Presence of infectious prions in urine}

Target questions 17 and 18 asked the experts to estimate the likelihood that infectious prion protein could be present in the urine of asymptomatic people with VCJD and CJD. In both cases, the experts indicated that prion protein could be present in urine of both vCJD and CJD patients, given the lengthy asymptomatic phase of these two diseases. Median estimates of the likelihood of infectious prions being present in urine were at $85 \%$ for asymptomatic vCJD cases and $15 \%$ for asymptomatic CJD cases.

\subsection{Transmission of prion disease through urine-derived fertility drugs}

Target questions 19-22 focused on transmission of prion disease via urine. Considering the current selection criteria for urine donors and current manufacturing processes (target questions 19 and 20), the expert group estimated the likelihood that infectious prions are present in urine-derived fertility drugs to be $1.4 \%$ for $\mathrm{vCJD}$ and $8 \%$ for CJD. These values reflect the smaller number of $\mathrm{VCJD}$ cases relative to CJD cases that could contaminate urine pools and the opinion those prions can co-purify during manufacturing of urine-derived fertility drugs.

Target questions 21 and 22 asked the experts to judge the likelihood that prion disease could be contracted through the use of urine-derived fertility drugs, due to the presence of infectious prions either from asymptomatic VCJD or CJD cases in human urine pools used to manufacture such drugs. In both cases, the risk of contracting prion disease via this route of transmission was estimated to be $1 \%$ by the experts, with $90 \%$ credible intervals ranging from $0 \%-11 \%$ in the case of urine samples from asymptomatic vCJD urine donors and $0-10 \%$ for asymptomatic CJD donors.

\subsection{Relative risk of urine-derived and recombinant fertility drugs}

Target questions 23-26 asked the experts to judge the relative risk of urine-derived and recombinant fertility drugs. Target questions 23 and 24 asked the experts to judge the likelihood that prion disease could be contracted through the use of fertility drugs 
manufactured using recombinant methods, either without or with the use of fetal bovine serum. The median values of $<10^{-6} \%$ (less than one in 100 million) and $<10^{-7} \%$ (less than one in a billion) reveal that the experts placed these at a much lower risk for prion disease transmission through the use of recombinant fertility drugs than urine-derived fertility drugs.

Table 2 Distribution of expert rating of effectiveness of strategies for managing the risk of prion disease from urine-derived fertility drugs

\begin{tabular}{|c|c|c|c|c|c|c|}
\hline \multicolumn{2}{|c|}{ Strategy } & \multirow{2}{*}{$\begin{array}{c}\begin{array}{c}\text { Not at all } \\
\text { effective }\end{array} \\
87.5\end{array}$} & \multirow{2}{*}{$\begin{array}{c}\text { Minimally } \\
\text { effective }\end{array}$} & \multirow{2}{*}{$\begin{array}{c}\begin{array}{c}\text { Moderately } \\
\text { effective }\end{array} \\
12.5\end{array}$} & \multirow{2}{*}{$\begin{array}{c}\begin{array}{c}\text { Very } \\
\text { effective }\end{array} \\
0.0\end{array}$} & \multirow{2}{*}{$\begin{array}{c}\begin{array}{c}\text { Extremely } \\
\text { effective }\end{array} \\
0.0\end{array}$} \\
\hline 1 & $\begin{array}{l}\text { Donor } \\
\text { selection/sourcing } \\
\text { from countries } \\
\text { without CJD }\end{array}$ & & & & & \\
\hline 2 & $\begin{array}{l}\text { Donor } \\
\text { selection/sourcing } \\
\text { based on time spent in } \\
\text { the UK }\end{array}$ & 12.5 & 32.5 & 41.3 & 13.8 & 0.0 \\
\hline 3 & $\begin{array}{l}\text { Donor } \\
\text { selection/sourcing } \\
\text { based on blood } \\
\text { transfusion, } \\
\text { neurosurgery, or } \\
\text { exposure to growth } \\
\text { hormone }\end{array}$ & 15.0 & 31.3 & 26.3 & 12.5 & 15.0 \\
\hline 4 & $\begin{array}{l}\text { Implementing a } \\
\text { validated process for } \\
\text { prion sterilisation of } \\
\text { urine pools }\end{array}$ & 12.5 & 12.5 & 23.8 & 21.3 & 30.0 \\
\hline 5 & $\begin{array}{l}\text { Testing for PrP in } \\
\text { urine pools used to } \\
\text { manufacture } \\
\text { gonadotropins }\end{array}$ & 75.0 & 12.5 & 12.5 & 0.0 & 0.0 \\
\hline 6 & $\begin{array}{l}\text { Testing for } \operatorname{PrP} \text { in the } \\
\text { final product }\end{array}$ & 62.5 & 0.0 & 6.3 & 16.3 & 15.0 \\
\hline 7 & $\begin{array}{l}\text { Banning the use of } \\
\text { urine-derived fertility } \\
\text { products }\end{array}$ & 12.5 & 12.5 & 0.0 & 2.5 & 72.5 \\
\hline 8 & $\begin{array}{l}\text { Banning the use of } \\
\text { recombinant fertility } \\
\text { products }\end{array}$ & 100.0 & 0.0 & 0.0 & 0.0 & 0.0 \\
\hline 9 & $\begin{array}{l}\text { Testing for } \mathrm{PrP}^{\mathrm{Sc}} \\
\text { using a sensitive, } \\
\text { validated test in urine } \\
\text { donor }\end{array}$ & 0.0 & 11.7 & 31.7 & 45.0 & 11.7 \\
\hline 10 & $\begin{array}{l}\text { Testing for } \mathrm{PrP}^{\mathrm{Sc}} \\
\text { using a sensitive, } \\
\text { validated test in final } \\
\text { product }\end{array}$ & 0.0 & 5.0 & 15.0 & 31.7 & 48.3 \\
\hline
\end{tabular}


Finally, target questions 25 and 26 focused on the expert group's judgment of the relative risk of contracting prion disease from urine-derived fertility drugs, as compared to fertility drugs manufactured using recombinant methods either with or without fetal bovine serum. The experts judged recombinant fertility drugs produced with bovine serum to possess 10 -fold lower risk, compared to urine-derived fertility drugs. Fertility drugs made without fetal bovine serum were judged to present a risk about 1,200 times less compared to urine-derived counterparts. The experts' responses to these last two target questions indicated their collective view that the risk of contracting prion disease from fertility drugs derived from human urine was higher than the risk from fertility drugs made using recombinant methods either with or without the use of fetal bovine serum. Further details on the results for target questions 1-26 are provided in the supplementary material (available at: https://dataverse.harvard.edu/dataset.xhtml? persistentId=doi\%3A10.7910\%2FDVN\%2FREVVTB\#).

\subsection{Strategy ranking}

This simple and easily implemented exercise generated semi-quantitative rankings regarding the effectiveness of the potential risk management strategies for prion disease contracted from taking urine-derived fertility drugs. The results of the strategy ranking exercise, in which seven experts ranked the perceived effectiveness of ten strategies for managing the potential risks of prion disease from urine-derived fertility drugs, are listed in Table 2. While most strategies showed unimodal shape with respect to the distribution of expert opinion (including strategies 2, 3, 4, 5, 8, 9 and 10), three strategies exhibited a bimodal distribution (strategies 1, 6 and 7), (the reasons for this bimodal pattern of expert opinion are unclear at this stage and warrant further investigation). All experts agreed that strategy number 8 - banning the use of recombinant fertility drugs - would be ineffective. The experts considered banning the use of urine-derived fertility drugs and screening such drugs for the presence of infectious prion protein with a sensitive and validated $\mathrm{PrP}^{\mathrm{Sc}}$ test to be the two most effective risk reduction strategies.

\section{Discussions}

In review of target questions 1-16 concerning the prevalence of asymptomatic prion disease in the general population, it is important to highlight that, according to the judgements of the experts, the prevalence of asymptomatic vCJD in the general population in North America is very low. This result concurs with surveillance data from the US Centers for Disease Control and Prevention (CDC) and Public Health Agency of Canada (PHAC) showing only three potential vCJD cases in the US and one case in Canada (a Saskatchewan immigrant from the UK) at the time the elicitation was conducted. With regard to the coherence of expert responses to the questions, the responses demonstrated that one or more experts had markedly different views from those of the rest of the panel. The discrepancies seem to involve a single expert, though not always the same expert. As it would seem unlikely that one expert had unique information not available to the other experts, these discrepancies would seem to reflect differences of expert judgement in the face of scientific uncertainty.

In the case of target questions 17 and 18, regarding the presence of infectious prions in urine and urine pools used in the manufacture of fertility drugs, the aggregated experts' 
responses indicate concurrence that there is a potential risk of disease associated with prion protein being present in the urine of asymptomatic VCJD and CJD patients. In both questions, one expert gave likelihoods that were effectively zero for these possibilities, whereas others ascribed real non-zero probabilities. Again, such differences may be attributed to differences in expert judgements in the absence of clear evidence on which to base more firm conclusions.

Target questions 19-22 dealt with the transmission of prion disease through the use of urine-derived fertility drugs. The pooled judgements indicated that there is potential risk that prion disease could be contracted through the use of fertility drugs derived from human urine contaminated with infectious prion proteins. The elicitation suggested $1 \%$ likelihood that prion disease could be contracted by using fertility drugs manufactured from human urine containing infectious prions of asymptomatic vCJD cases (questions 21 and 22). According to verbal risk scales, reviewed by Calman and Royston (1997), a median 1\% or 1-in-100 value is considered to be 'high risk', well above de minimis risk levels of 1 in a million used by regulators when assessing acceptable risk (Calman and Royston, 1997). The range graph results from these questions (see the supplementary material) also showed wide-ranging individual uncertainties. For some of these elicited questions, one expert ascribed a much higher likelihood to the possibility of such a scenario than the rest of the group, while another expert was inclined to express maximal uncertainty through their elicited response. Such responses are not fallacious or irrational, but signal the real challenge of trying to pin down meaningful likelihoods when data are sparse or non-existent; this situation should be communicated to decision takers.

The likelihood that prion disease could be contracted through the use of fertility drugs manufactured using alternative recombinant methods (target questions 23 and 24) were considered to be negligible (at or below de minimis risk levels). However, the views of one or two experts again deviated from the rest of the group. The relative risks of contracting prion disease from urine-derived fertility drugs, as compared to fertility drugs manufactured using recombinant methods with or without fetal bovine serum (target questions 25 and 26), produced wide-ranging estimates, hinting at a possible dichotomy of views on this topic. Disaggregation and further discussion of the factors involved might help elucidate the issues better and identify potential knowledge gaps. This last remark underlines a potential valuable benefit of conducting a structured elicitation exercise: the expert judgments and the demonstration quantitatively of the existence of major uncertainties will provoke new lines of thinking and prompt ideas about research topics that could be pursued.

Rather than a fulsome, evidence-based risk assessment, the expert elicitation presented here should be regarded as provisional, subject to modification as new evidence becomes available. The elicitation results may also provide a basis for identifying that significant knowledge gaps exist and for prioritising issues that might be amenable to further targeted research.

From the present expert elicitation, nearly all target questions have large credible intervals associated with them, indicating a high degree of uncertainty in relation to the questions posed. This is interpreted not as a failure of the elicitation, but as demonstration of the extent of genuine scientific uncertainty that presently exists with respect to the many challenging issues relating to prion infection processes and risk factors. However, the credible intervals obtained by applying weights from the performance-based analysis are generally smaller than those calculated with unweighted pooling. This reflects the fact 
that experts, whose judgements were better validated statistically on the seed questions, generally expressed lower judgement uncertainties on the target items.

In addition to expressing their judgements about the potential risks of prion disease associated with the use of fertility drugs, the experts also provided their views on strategies that might be considered to manage potential risks of this type. The least effective strategies were strategy 1 (donor selection/sourcing from countries without CJD; $87.5 \%$ not at all effective) and strategy 8 (banning the use of recombinant fertility products; $100 \%$ not at all effective). Strategy 1 focuses on restricting urine collection to countries without CJD; although this may appear, at first, to be an attractive option, it would be difficult to implement in practice as there are few countries without reported CJD. The expert group was aware of this fact and ranked this strategy as one of the least effective options.

In contrast, strategy 7 (banning the use of urine-derived fertility products; $72.5 \%$ extremely effective), followed by strategy 10 (testing the final product with a sensitive and validated test for $\operatorname{PrP}^{\mathrm{Sc}} ; 48.3 \%$ extremely effective), were ranked as the two most effective strategies for managing prion disease risk. While a ban on urine-derived fertility products might be seen as prudent from a precautionary point of view, both the benefits and risks of fertility drugs and indeed any therapeutic product need to be carefully considered throughout the entire life cycle of that product (Institute of Medicine, 2012).

\section{Conclusions}

The present expert elicitation to explore the areas of uncertainty related to prion transmission and urine-derived pharmaceuticals was undertaken at a specific point in time (specifically in October, 2011). After the expert elicitation meeting, the Canadian Fertility and Andrology Society (CFAS) and the European Society of Human Reproduction and Embryology (ESHRE) assessed the data from Van Dorsselaer et al. (2011), stating that the concern raised by the authors was not substantiated by clinical evidence and a causal relationship between urine-derived fertility drugs and prion disease had not been demonstrated. It should be emphasised that Van Dorsselaer et al. (2011) detected the normal prion form of the prion protein $\left(\mathrm{PrP}^{\mathrm{C}}\right)$ in urine-derived fertility drugs and not infectious prion protein $\left(\operatorname{PrP}^{\mathrm{Sc}}\right)$. The fertility societies point to the 'absence of evidence' of prion disease risk, stating that urine-derive gonadotropins have been used for over 50 years without reports of prion disease transmission (CFAS Prion Position Statement, 2011; ESHRE, 2012). However, this should be qualified by the fact that only some countries have set up national CJD and vCJD surveillance systems post-1994, after the detection of the first cases of VCJD in the UK. Thus, formal surveillance for CJD and vCJD by all potential routes (including urine-derived transmission) has been in place in most countries for only about two decades. The fertility societies found the clinical significance of the data from Van Dorsselaer et al. (2011) difficult to assess, but remain vigilant in reviewing and evaluating new scientific data.

For human prion disease there is a difference between the level of risk when comparing VCJD to SCJD, as reported in the scientific literature. In SCJD patients, small quantities of $\operatorname{PrP}^{\mathrm{Sc}}$ have been identified in peripheral organs. Research published after the expert elicitation, conducted by Notari et al. (2012), investigated infectivity of urine from patients with SCJDMM1, the most common subtype of SCJD; it was found not to contain detectable prion infectivity as determined by bioassay and suggests that no significant 
prionuria occurs in this common subtype of human prion disease. Based on this study, the occurrence of infectious prions in SCJD patients' urine appears to be remote. It was further demonstrated that normal urine contains discrete amounts of the C-terminal fragment of PrP, matching the $\mathrm{C} 1$ fragment, but not full-length PrP. Although $\mathrm{C} 1$ may not be a good substrate for $\mathrm{PrP}^{\mathrm{Sc}}$ replication it may act as modulator to slow the accumulation of neurotoxic forms of PrP (Dagdanova et al., 2010; Westergard et al., 2011).

Rubenstein and Chang (2013) found $\mathrm{PrP}^{\mathrm{Sc}}$ in non-CNS human tissues (spleens, lymph nodes and tonsils) from sCJD individuals (Rubenstein and Chang, 2013). Using protein misfolding cyclic amplification (PMCA) followed by a sensitive immunoassay (surround optical fibre immunoassay, SOFIA), $\operatorname{PrP}^{\mathrm{Sc}}$ was detected in the cerebrospinal fluid (CSF) but neither in urine nor blood of SCJD patients. More recently, published findings show that the blood (plasma fraction) of SCJD patients does contain infectivity that transmits transmissible spongiform encephalopathy (TSE) disease to experimental animals with an infectivity level similar to that of vCJD (Douet et al., 2014). It is important for public health and other stakeholders to be vigilant as research continues to explore the complexity of prion biology uncertainties.

Some studies have demonstrated that prions can be found in the urine excreted by scrapie-inoculated laboratory mice and hamsters. Results from these studies also found that the infectious urines are excreted from infected animals during both the asymptomatic incubation period before the appearance of any clinical signs (Seeger et al., 2005) and during the symptomatic phase (Seeger et al., 2005; Kariv-Inbal et al., 2006). The titer of infectious prions in the urine of scrapie-infected hamsters is comparable to that found in their blood (Gregori et al., 2008).

Prion proteins were detected in human urine-derived gonadotropins (Kuwabara et al., 2009), but not in the recombinant human gonadotropins (Van Dorsselaer et al., 2011). The transmission of CJD through urine has only been demonstrated in a mouse model by intracerebral inoculation (Tateishi, 1985). Again, it must be emphasised that no human case of urinary transmission of CJD has been reported to date.

In vCJD patients, younger individuals are affected (median age at death of 28 years) compared to SCJD which affects older individuals (median age at death of 68 years). $\mathrm{PrP}^{\mathrm{Sc}}$ is found in the brain and in non-CNS human tissues (spleens, lymph nodes and tonsils) of vCJD patients. The peripheral $\mathrm{PrP}^{\mathrm{Sc}}$ levels in $\mathrm{SCJD}$ and $\mathrm{vCJD}$ individuals were found not to differ significantly and as anticipated, were several logs lower than amounts found in brain (Rubenstein and Chang, 2013).

Following the completion of the expert elicitation reported here, Moda et al. (2014) reported finding infectious prion proteins in urine samples from vCJD patients. Specifically, $\operatorname{PrP}^{\mathrm{Sc}}$ was detected in 13 of 14 urine samples from patients with $\mathrm{vCJD}$, but not in urine samples from $68 \mathrm{sCJD}$ patients, 4 patients with genetic CJD patients, 50 patients with other neurodegenerative disorders, or 50 healthy controls. Although the positive samples contained only minute quantities of $\mathrm{PrP}^{\mathrm{Sc}}$, the detection of infectious prions only in urine samples from vCJD patients suggests that urine from vCJD patients could be uniquely affected within the CJD family of diseases.

There are lengthy incubation times for human prion diseases and the existence of subclinical infections in M129M, M129V and V129V PRNP carriers that increases the uncertainty surrounding the future trajectory of vCJD infections which has implications for the management of blood, blood products and handling of surgical instruments (Gill et al., 2013; Peden et al., 2004). 
Sourcing of urine pools is considered to be an important TSE risk reduction factor. A 2007 European Medicines Agency expert panel reported the TSE risk linked to gonadotrophins derived from the urine of post-menopausal Argentine women can be considered a negligible risk for a number of reasons including:

1 Argentina's negligible BSE risk (GBR I) status

2 the absence of $\mathrm{VCJD}$ cases

3 the selection of donors with no known TSE risk

4 the theoretical TSE removal capacity of the manufacturing process.

Moreover, women treated with fertility drugs are injected by subcutaneous routes of administration, which is known to be a relatively inefficient route for transmitting TSEs (EMAR, 2009).

During the present elicitation, the experts indicated that there was a possibility that prion disease could be transmitted through the use of fertility medications derived from urine contaminated with infectious prions from asymptomatic CJD cases. However, the evidence discussed previously suggests a greater concern about urine-derived from vCJD rather than $\mathrm{SCJD}$ cases.

Not only is the risk profile associated with the transmission via urine for VCJD different to SCJD it highlights that there is also a differing geographic risk profile associated with vCJD.

Due to specified risk material (SRM) bans in many affected countries, new cases of vCJD by consuming contaminated meat products have sharply declined (Bradley et al., 2006). A recent British survey has estimated that approximately 1 in 2,000 people in the population may be silent carriers of $\mathrm{VCJD}$ based on detecting prion protein in their lymphorecticular tissue (Gill et al., 2013). vCJD has been documented to be transmissible by blood as there are four known cases in the UK where patients received blood transfusion from pre-symptomatic donors and subsequently developed clinical vCJD (Llewelyn et al., 2004; Peden et al., 2004), showing that people can contract vCJD and incubate as asymptomatic carriers by means other than consuming infected bovine products. The risk of asymptomatic carriers was first highlighted when low levels of infectivity were detected in blood components of mice infected with a human-derived strain of vCJD during both the preclinical and clinical phases of the disease (Cervenakova et al., 2003). Thus, for some areas, such as the UK and France, transmission of vCJD by silent carriers may present more concern for disease transmission through blood or other blood products sourced from humans in those countries.

Although there is no known case for contracting prion diseases through the administration of urine-derived human gonadotropins, the widespread use of these drugs by over 300,000 women in the US and Canada for infertility treatment makes it a potential health risk to the public. Indeed, there were precedents in Australia where four known cases of CJD occurred, transmitted by taking fertility drugs derived from human pituitary hormone (gonadotropins) (Boyd et al., 2001). In addition, the purification procedures used to isolate therapeutic gonadotropins from human urine may also enrich the prion proteins, as demonstrated by close to 200 cases of CJD infection through intramuscular injections of pituitary growth hormone-derived from human cadavers in the 1990s (Brown et al., 2006). In some French batches of these pituitary growth hormones, 
the recipient CJD disease rates were reported to exceed $10 \%$, which highlighted the high infectivity of parenterally-injected prions present in some gonadotropin preparations.

The findings that normal and infectious prion protein can be found in human urine (Moda et al., 2014; Van Dorsselaer et al., 2011) may warrant a reassessment of whether or not the risks of urine-derived fertility drugs may outweigh their benefits. The judgment of whether the outcomes of the present elicitation exercise are helpful in this regard is ultimately a matter for regulatory authorities responsible for evaluating public health issues relating to fertility drugs and prion diseases to decide.

While a formal assessment of the likelihood of prion disease transmission through the use of urine-derived fertility drugs is impossible due to a current lack of relevant scientific data, the structured expert elicitation provided a useful means of addressing prion disease risk uncertainties associated with urine-derived fertility drugs, thereby serving to characterise the scientific uncertainty surrounding this issue (Institute of Medicine, 2014). The use of recombinant gonadotropins was also taken into consideration during the expert elicitation process as a viable alternative, which does not require extraction from human urine. Although the present expert elicitation judged recombinant fertility drugs to carry relatively less risk than urine-derived fertility drugs, expert opinions will continue to evolve as new scientific information of the type summarised herein becomes available.

\section{References}

Aspinall, W. (2010) 'A route to more tractable expert advice', Nature, Vol. 63, No. 7279, pp.294-295.

Boyd, A., Fletcher, A., Lee, J.S., Lewis, V., Masters, C.L. and Collins, S.J. (2001) 'Transmissible spongiform encephalopathies in Australia', Communicable Diseases Intelligence Quarterly Report, Vol. 25, No. 4, pp.248-252.

Bradley, R., Collee, J.G., Liberski, P.P. (2006) 'Variant CJD (vCJD) and bovine spongiform encephalopathy (BSE): 10 and 20 years on: part 1', Folia Neuropathologica, Vol. 44, No. 2, pp.93-101.

Brown, P. (2001) 'Bovine spongiform encephalopathy and variant Creutzfeldt-Jakob disease', $B M J$, Vol. 322, No. 7290, pp.841-844.

Brown, P., Brandel, J.P., Preece, M. and Sato, T. (2006) 'Iatrogenic Creutzfeldt-Jakob disease: the waning of an era', Neurology, Vol. 67, No. 3, pp.389-393.

Bruce, M.E. (2000) 'New variant' Creutzfeldt-Jakob disease and bovine spongiform encephalopathy', Nature Medicine, Vol. 6, No. 3, pp.258-259.

Calman, K.C and Royston, G.H. (1997) 'Risk language and dialects', BMJ, Vol. 315, No. 7113, pp.939-942.

Cervenakova, L., Yakovleva, O., McKenzie, C., Kolchinsky, S., McShane, L., Drohan, W.N. and Brown, P. (2003) 'Similar levels of infectivity in the blood of mice infected with human-derived vCJD and GSS strains of transmissible spongiform encephalopathy', Transfusion, Vol. 43, No. 12, pp.1687-1694.

CFAS Prion Position Statement (2011) [online] https://cfas.ca/prion-position-statement-2/ (accessed 15 June 2016).

Chen, C.C., Wang, Y.H. and Wu, K.Y. (2013) 'Consumption of bovine spongiform encephalopathy (BSE) contaminated beef and the risk of variant Creutzfeldt-Jakob disease', Risk Analysis, Vol. 33, No.11, pp.1958-1968.

Cooke R.M. (1991) Experts in Uncertainty: Opinion and Subjective Probability in Science, Oxford University Press, New York. 
Cooke, R.M and Goossens, L.H.J. (2008) 'TU Delft expert judgment data base', Reliability Engineering and System Safety, Vol. 93, No. 5, pp.657-674.

Croes, E.A., Roks, G., Jansen, G.H., Nijssen, P.C. and van Duijn, C.M. (2002) 'Creutzfeldt-Jakob disease 38 years after diagnostic use of human growth hormone', Journal of Neurology, Neurosurgery and Psychiatry, Vol. 72, No. 6, pp.792-793.

Dagdanova, A., Ilchenko, S., Notari, S., Yang, Q., Obrenovich, M.E., Hatcher, K., McAnulty, P., Huang, L., Zou, W., Kong, Q., Gambetti P. and Chen, S.G. (2010) 'Characterization of the prion protein in human urine', Journal of Biological Chemistry, Vol. 285, No. 40, pp.30489-30495.

Douet, J.Y., Zafar, S., Perret-Liaudet, A., Lacroux, C., Lugan, S., Aron, N., Cassard, H., Ponto, C., Corbière, F., Torres, J.M., Zerr, I. and Andreoletti, O. (2014) 'Detection of infectivity in blood of persons with variant and sporadic Creutzfeldt-Jakob disease', Emerging Infectious Diseases, Vol. 20, No. 1, pp.114-117.

ESHRE (2012) ESHRE Position Statement Concerning Prion Detection in Urinary Gonadotropin Formulations [online] http://transmissiblespongiformencephalopathy.blogspot.ca/2012/01/ eshre-position-statement-concerning.html (accessed 15 June 2016).

European Medicines Agency Report (EMAR, 2009) Expert Workshop on CJD Risk and Urine-Derived Medicinal Products, EMEA, 12-13 July 2007, London [online] http://www.ema.europa.eu/docs/en GB/document library/Report/2009/11/WC50001539 4.pdf (accessed 15 June 2016).

Gill, O.N., Spencer, Y., Richard-Loendt, A., Kelly, C., Dabaghian, R., Boyes, L., Linehan, J., Simmons, M., Webb, P., Bellerby, P., Andrews, N., Hilton, D.A., Ironside, J.W., Beck, J., Poulter, M., Mead, S. and Brandner, S. (2013) 'Prevalent abnormal prion protein in human appendixes after bovine spongiform encephalopathy epizootic: large scale survey', $B M J$, Vol. 347, No. f5675, DOI: http://dx.doi.org/10.1136/bmj.f5675.

Gough, K.C. and Maddison, B.C. (2010) 'Prion transmission: prion excretion and occurrence in the environment', Prion, Vol. 4, No. 4, pp.275-282.

Gregori, L., Kovacs, G.G., Alexeeva, I., Budka, H. and Rohwer, R.G. (2008) 'Excretion of transmissible spongiform encephalopathy infectivity in urine', Emerging Infectious Diseases, Vol. 14, No. 9, pp.1406-1412.

Hamaguchi, T., Noguchi-Shinohara, M., Nozaki, I., Nakamura, Y., Sato, T., Kitamoto, T., Mizusawa, H. and Yamada, M. (2009) 'The risk of iatrogenic Creutzfeldt-Jakob disease through medical and surgical procedures', Neuropathology, Vol. 29, No. 5, pp.625-631.

Hueston, W.D. (2013) 'BSE and variant CJD: emerging science, public pressure and the vagaries of policy-making', Preventive Veterinary Medicine, Vol. 109, Nos. 3-4, pp.179-184.

Institute of Medicine (2012) Ethical and Scientific Issues in Studying the Safety of Approved Drugs, National Academies Press, Washington, DC.

Institute of Medicine (2014) Characterizing and Communicating Uncertainty in the Assessment of Benefits and Risks of Pharmaceutical Products: Workshop Summary, National Academies Press, Washington, DC.

Kariv-Inbal, Z., Ben-Hur, T., Grigoriadis, N.C., Engelstein, R. and Gabizon, R. (2006) 'Urine from scrapie-infected hamsters comprises low levels of prion infectivity', Neurodegenerative Disease, Vol. 3, No. 3, pp.123-128.

Kuwabara, Y., Mine, K., Katayama, A., Inagawa, T., Akira, S. and Takeshita, T. (2009) 'Proteomic analyses of recombinant human follicle -stimulating hormone and urinary-derived gonadotropin preparations', Journal of Reproductive Medicine, Vol. 54, No. 8, pp.459-466.

Llewelyn, C.A., Hewitt, P.E., Knight, R.S., Amar, K., Cousens, S., Mackenzie, J. and Will, R.G. (2004) 'Possible transmission of variant Creutzfeldt-Jakob disease by blood transfusion', Lancet, Vol. 363, No. 9407, pp.417-421. 
Moda, F., Gambetti, P., Notari, S., Concha-Marambio, L., Catania, M., Park, K.W., Maderna, E., Suardi, S., Haik, S., Brandel, J.P., Ironside, J., Knight, R., Tagliavini, F. and Soto, C. (2014) 'Prions in the urine of patients with variant Creutzfeldt-Jakob disease', New England Journal of Medicine, Vol. 371, No. 6, pp.530-539.

Notari, S., Qing, L., Pocchiari, M., Dagdanova, A., Hatcher, K., Dogterom, A., Groisman, J.F., Lumholtz, I.B., Puopolo, M., Lasmezas, C., Chen, S.G., Kong, Q. and Gambetti, P. (2012) 'Assessing prion infectivity of human urine in sporadic Creutzfeldt-Jakob disease', Emerging Infectious Diseases, Vol. 18, No. 1, pp.21-28.

Peden, A.H., Head, M.W., Ritchie, D.L., Bell, J.E. and Ironside, J.W. (2004) 'Preclinical vCJD after blood transfusion in a PRNP codon 129 heterozygous patient', Lancet, Vol. 364, No. 9433, pp.527-529.

Prusiner, S.B. (1998) 'Prions', Proceedings of the National Academy of Sciences of the United States of America, Vol. 95, No. 23, pp.13363-13383.

Puopolo, M., Ladogana, A., Vetrugno, V. and Pocchiari, M. (2011) 'Transmission of sporadic Creutzfeldt-Jakob disease by blood transfusion: risk factor or possible biases', Transfusion, Vol. 51, No. 7, pp.1556-1566.

Reichl, H., Balen, A. and Jansen, C.A. (2002) 'Prion transmission in blood and urine: what are the implications for recombinant and urinary-derived gonadotrophins?', Human Reproduction, Vol. 17, No. 10, pp.2501-2508.

Rubenstein, R and Chang, B. (2013) 'Assessment of $\operatorname{PrP}^{\mathrm{Sc}}$ distribution in sporadic and variant CJD’, PLoS One, Vol. 8, No. 7, p.e66352.

Seeger, H., Heikenwalder, M., Zeller, N., Kranich, J., Schwarz, P., Gaspert, A., Seifert, B., Miele, G. and Aguzzi, A. (2005) 'Coincident scrapie infection and nephritis lead to urinary prion excretion', Science, Vol. 310, No. 5746, pp.324-326.

Sikorska, B., Knight, R., Ironside , J.W. and Liberski, P.P. (2012) 'Creutzfeldt-jakob disease', in Neurodegenerative Diseases, Vols. 76-90. Springer, USA.

Tateishi, J. (1985) 'Transmission of Creutzfeldt-Jakob disease from human blood and urine into mice', Lancet, Vol. 2, No. 8463, p.1074.

Tyshenko, M.G., ElSaadany, S., Oraby, T., Darshan, S., Aspinall, W., Cooke, R., Catford, A. and Krewski, D. (2011) 'Expert elicitation for the judgment of prion disease risk uncertainties', Journal of Toxicology and Environmental Health, Part A, Vol. 74, Nos. 2-4, pp.261-285.

Tyshenko, M.G., ElSaadany, S., Oraby, T., Darshan, S., Catford, A., Aspinall, W., Cooke, R. and Krewski, D. (2012) 'Expert judgment and re-elicitation for prion disease risk uncertainties', International Journal of Risk Assessment and Management, Vol. 16, Nos.1-3, pp.48-77.

Van De Weijer, B.H., Mulders, J.W., Bos, E.S., Verhaert, P.D. and van den Hooven, H.W. (2003) 'Compositional analyses of a human menopausal gonadotrophin preparation extracted from urine (menotropin). Identification of some of its major impurities', Reproductive BioMedicine Online, Vol. 7, No. 5, pp.547-557.

Van Dorsselaer, A., Carapito, C., Delalande, F., Schaeffer-Reiss, C., Thierse, D., Diemer, H., McNair, D.S., Krewski, D. and Cashman, N.R. (2011) 'Detection of prion protein in urine-derived injectable fertility products by a targeted proteomic approach', PLoS One, Vol. 6, No. 3, p.e17815.

Westergard, L., Turnbaugh J.A. and Harris D.A. (2011) 'A naturally occurring C-terminal fragment of the prion protein $(\mathrm{PrP})$ delays disease and acts as a dominant-negative inhibitor of $\operatorname{PrP}^{\mathrm{Sc}}$ formation', Journal of Biological Chemistry, Vol. 286, No. 51, pp.44234-4442.

Wolfenson, C., Groisman, J., Couto, A.S., Hedenfalk, M., Cortvrindt, R.G., Smitz, J.E. and Jespersen, S. (2005) 'Batch-to-batch consistency of human-derived gonadotrophin preparations compared with recombinant preparations', Reproductive BioMedicine Online, Vol. 10, No. 4, pp.442-454. 